Research Paper

\title{
Analysis of MEDI 2 Mutation in Multiple Uterine Leiomyomas in South Korean patients
}

\author{
Minkyoung Lee*, Keunyoung Cheon*, Boah Chae, Hyesung Hwang, Hyun-Kyung Kim, Youn-Jee Chung, \\ Jae-Yen Song, Hyun-Hee Cho, Jang-Heub Kim, Mee-Ran Kim ${ }^{凶}$ \\ Department of Obstetrics and Gynecology, College of Medicine, The Catholic University of Korea, 222, Banpo-daero, Seocho-gu, Seoul, 06591, Republic of Korea \\ *Equally contributed. \\ $\triangle$ Corresponding author: Mee-Ran Kim, Department of Obstetrics and Gynecology, College of Medicine, The Catholic University of Korea, 222, Banpo-daero, \\ Seocho-gu, Seoul, 06591, Republic of Korea Tel: +82-2-2258-6170, Fax: +82-2-595-1549, E-mail: mrkim@catholic.ac.kr \\ (c) Ivyspring International Publisher. This is an open access article distributed under the terms of the Creative Commons Attribution (CC BY-NC) license \\ (https://creativecommons.org/licenses/by-nc/4.0/). See http://ivyspring.com/terms for full terms and conditions.
}

Received: 2017.07.10; Accepted: 2017.10.30; Published: 2018.01.01

\begin{abstract}
Uterine leiomyomas are one of the most common benign gynecologic tumors, but the exact causes are not completely understood. In 2011, through DNA sequencing, MEDI 2 mutation was discovered in approximately $71 \%$ of uterine leiomyomas. Several recent studies confirmed the high frequency of MED 12 mutation in uterine leiomyoma. Nevertheless, no study has been done on MED 12 mutation in the case of patients with multiple leiomyomas in a patient. The purpose of this study was to investigate the frequency of MEDI 2 mutations in uterine leiomyomas of South Korean patients. In addition, we examined MED 12 mutation in multiple leiomyomas in the same patients.

Uterine leiomyoma tissues were obtained from symptomatic women who underwent hysterectomy or myomectomy for medically indicated reasons. We collected 60 uterine leiomyomas from 41 women. Tumor size ranged from 1 to $12 \mathrm{~cm}$. Patients' ages ranged from 25 to 55 years with an average of 38.4 years.

Of the 60 tumors, 40 (66.67\%) displayed MED 12 mutation. Among the 41 patients, 14 patients had multiple leiomyomas and we analyzed those multiple leiomyomas. Three of them had the same mutations. Five of them, each leiomyoma had a different mutation. Two of them did not have mutation. Four of them had both mutation-positive and mutation-negative leiomyomas.

In conclusion, we confirmed the high frequency of the MED 12 mutation in uterine leiomyomas of South Korean patients. We also identified various MED 12 mutation status in patients with multiple leiomyomas. This suggests that in a given patient, different tumors may have arisen from different cell origins and therefore it is supposed that occurrence of multiple leiomyoma in a single patient may not be caused by intrauterine metastasis or dissemination.
\end{abstract}

Key words: MED12, uterine leiomyoma, somatic mutation, multiple leiomyoma.

\section{Introduction}

Uterine leiomyomas, also known as fibroids are the most common gynecological neoplasm in women of reproductive age. Recently, uterine leiomyomas are increasing in nulliparous women due to delayed marriage and delivery. It also causes female infertility, abnormal uterine bleeding, dysmenorrhea, and pelvic pain [1].

Unfortunately, the exact causes of uterine leiomyomas are not completely understood. Nulliparity, early menarche, late menopause and obesity increase the size of lesions while the tumors usually shrink after menopause, which supports estrogen and progesterone are important regulators of leiomyoma growth [2].

In recent studies, several chromosomal aberrations have been observed in approximately $40 \%-50 \%$ of uterine leiomyomas, such as deletions of $7 \mathrm{q}$ and rearrangements involving $12 \mathrm{q} 15$ and $6 \mathrm{p} 21$. These occur in approximately $17 \%, 20 \%$, and $5 \%$ of karyotypically abnormal lesions, respectively [3, 4]. 
In addition to chromosomal change, Makinen et al. recently examined uterine leiomyoma tissues by exome sequencing and identified somatic mutations of mediator complex subunit 12 (MED12). They discovered mutations in MED12 exon 2 in 159 of 225 uterine leiomyomas (71\%). MED12 is a subunit of the Mediator complex that regulates transcription via RNA polymerase II [5]. Both missense and inframe insertion-deletion mutations have been reported, with codon 44 being the most prevalent mutational hotspot $(39 \%-96 \%)[6,7]$. The frequency of MED12 mutations has been reported to vary $50 \%$ to $80 \%$, depending on the ethnicity of examined patients $[6,8,9]$.

In the study of Makinen et al. no tumor displayed more than one mutation [5]. And former studies did not describe the MED12 mutation status of multiple leiomyoma in one patient. The purpose of this study was to investigate the frequency of MED12 mutations in uterine leiomyoma of South Korean patients to confirm that MED12 had a major role in the pathogenesis of uterine leiomyoma. In addition, we examined MED12 mutation in multiple leiomyomas in the same patients.

\section{Materials and methods}

\section{Tissue samples and DNA extraction}

Uterine leiomyoma tissues were obtained from symptomatic women who underwent hysterectomy or myomectomy for medically indicated reasons at Seoul St. Mary's Hospital. We collected 60 uterine leiomyomas from 41 women. Tumor size ranged from 1 to $12 \mathrm{~cm}$. Patients' age ranged from 25 to 55 years with an average of 38.4 years. The other basic characteristics of all enrolled patients are summarized in Table 1.

Table 1. Basic characteristics of the 41 enrolled patients

\begin{tabular}{ll}
\hline Variable & Data \\
\hline Age & $38.4 \pm 7.2^{*}$ \\
Body Mass Index & $22.6 \pm 9.0$ \\
Menarche & $13.8 \pm 1.3$ \\
Parity & $0.63 \pm 0.9$ \\
Married patients & $19(46.3 \%)^{* *}$ \\
Combined gynecologic disease & \\
Endometriosis & $12(29.1 \%)$ \\
Adenomyosis & $5(12.2 \%)$ \\
\hline${ }^{*}$ mean \pm SD; *number of patients (percentage)
\end{tabular}

\section{Genomic DNA extraction}

We used the Qiagen DNeasy Tissue kit (Qiagen, Hilden, Germany) and the protocols for fresh frozen tissues. Tissue samples were then lysed under denaturing conditions with a proteinase $\mathrm{K}$ digestion at $56^{\circ} \mathrm{C}$ for $3 \mathrm{~h}$. DNA was purified by column purification with a filter membrane and stored in $-20^{\circ} \mathrm{C}$ before use.

\section{MEDI 2 mutation analysis}

10 ng of genomic DNA extracted from each leiomyoma tissues was amplified by polymerase chain reaction (PCR). Primers used for the amplification of MED12 sequence were 5'-AACTAAACGCCGCTTTCCTG-3' (forward) and 5'-TTCCTTCAGCCTGGCAGAG-3' (reverse); product size: 159 base pairs. The PCR products were electrophoresed in a $2 \%$ agarose gel. Isolated PCR products were sequenced directly by Big Dye Terminator v.3.1 Cycle sequencing chemistry on a 96 capillary array DNA sequencer ABI 3730XL (Applied Biosystems, Foster City, CA, USA).

\section{Results}

Of all 60 leiomyomas, $66.67 \%(40 / 60)$ had MED12 mutations. All these point mutations were found in two nucleotide sites of c.130 and c.131. Six types of mutations were found and the most common mutation was c.130G>T (p.G44C) (Table 2).

Table 2. MED I 2 mutations in Uterine leiomyoma

\begin{tabular}{ll}
\hline MED12 mutation type & $\mathrm{N}=60$ \\
\hline c.130G $>$ A (p.G44S) & 7 \\
c.130G $>$ C (p.G44R) & 3 \\
c.130G $>$ T (p.G44C) & 11 \\
c.131G $>$ A (p.G44D) & 9 \\
c.131G $>$ C (p.G44A) & 6 \\
c.131G $>$ T (p.G44V) & 4 \\
No mutation & 20
\end{tabular}

Twenty-seven patients had single leiomyoma and average size of collected leiomyomas was 7.46 $\mathrm{cm}$. Fourteen patients had multiple leiomyomas. All 33 leiomyomas from 14 patients showed average size of $4.21 \mathrm{~cm}$ which was significantly smaller than the mean size of single leiomyoma $(p<0.05)$. Of 27 patients who have only one leiomyoma, 40.74\% showed no mutation while patients who have multiple leiomyoma showed no mutations in $27.27 \%$ (Table 3).

Table 3. Single versus multiple leiomyoma patient

\begin{tabular}{|c|c|c|c|}
\hline \multicolumn{2}{|c|}{ Patients with single leiomyoma } & \multicolumn{2}{|c|}{ Patients with multiple leiomyomas } \\
\hline MED12 mutation type & $\mathrm{N}=27$ & MED12 mutation type & $\mathrm{N}=33$ \\
\hline c.130G>A (p.G44S) & 2 & c.130G>A (p.G44S) & 5 \\
\hline c.130G>C (p.G44R) & 3 & c.130G >C (p.G44R) & 0 \\
\hline c.130G>T (p.G44C) & 4 & c.130G > T (p.G44C) & 7 \\
\hline c.131G>A (p.G44D) & 3 & c.131G>A (p.G44D) & 6 \\
\hline c.131G>C (p.G44A) & 4 & c.131G >C (p.G44A) & 2 \\
\hline c.131G>T (p.G44V) & 0 & c.131G>T (p.G44V) & 4 \\
\hline No mutation & $11(40.74 \%)$ & No mutation & $9(27.27 \%)$ \\
\hline Mean size $(\mathrm{cm})$ & 7.46 & Mean size $(\mathrm{cm})$ & 4.21 \\
\hline
\end{tabular}


We analyzed leiomyomas of patients who have multiple leiomyomas. Among 14 patients, mutations in each leiomyoma were identical in 3 patients $(\# 13$, \#14, \#34). And for 5 out of 14 patients, different mutations were found in each leiomyoma (\#11, \#36, $\# 38$, \#39, \#45). Two patients had no mutations in their leiomyomas (\#33, \#47). In 4 patients, some leiomyomas had mutations but other leiomyomas were mutation free (\#21, \#37, \#48, \#49) (Table 4).

Table 4. Various kinds of MEDI2 mutations in multiple leiomyomas

\begin{tabular}{|c|c|c|c|}
\hline Patients & Age & Size $(\mathrm{cm})$ & Mutation status of MED12 \\
\hline \multirow[t]{2}{*}{$\# 11$} & 42 & 1 & c.131G>T (p.G44V) \\
\hline & & 3 & c.130G>A (p.G44S) \\
\hline \multirow[t]{2}{*}{$\# 13$} & 30 & 3 & c.131G>A (p.G44D) \\
\hline & & 6 & c.131G>A (p.G44D) \\
\hline \multirow[t]{2}{*}{$\# 14$} & 31 & 1 & c.130G>T (p.G44C) \\
\hline & & 3 & c.130G>T (p.G44C) \\
\hline \multirow[t]{2}{*}{ \#21 } & 39 & 1 & c.130G>A (p.G44S) \\
\hline & & 1 & No mutation \\
\hline \multirow[t]{2}{*}{$\# 33$} & 39 & 2 & No mutation \\
\hline & & 8 & No mutation \\
\hline \multirow[t]{3}{*}{$\# 34$} & 25 & 1 & c.130G>T (p.G44C) \\
\hline & & 6 & c.130G>T (p.G44C) \\
\hline & & 9 & c.130G>T (p.G44C) \\
\hline \multirow[t]{2}{*}{$\# 36$} & 41 & 2 & c.130G>A (p.G44S) \\
\hline & & 6 & c.131G>A (p.G44D) \\
\hline \multirow[t]{2}{*}{$\# 37$} & 35 & 5 & c.130G>T (p.G44C) \\
\hline & & 8 & No mutation \\
\hline \multirow[t]{3}{*}{$\# 38$} & 33 & 2 & c.131G>A (p.G44D) \\
\hline & & 7 & c.131G>C (p.G44A) \\
\hline & & 11 & c.131G>T (p.G44V) \\
\hline \multirow[t]{2}{*}{ \#39 } & 30 & 2 & c.130G>T (p.G44C) \\
\hline & & 6 & c.131G>T (p.G44V) \\
\hline \multirow[t]{2}{*}{$\# 45$} & 34 & 3 & c.130G>A (p.G44S) \\
\hline & & 7 & c.131G>A (p.G44D) \\
\hline \multirow[t]{3}{*}{$\# 47$} & 33 & 1 & No mutation \\
\hline & & 3 & No mutation \\
\hline & & 7 & No mutation \\
\hline \multirow[t]{2}{*}{$\# 48$} & 41 & 1 & No mutation \\
\hline & & 4 & c.130G>A (p.G44S) \\
\hline \multirow[t]{3}{*}{$\# 49$} & 40 & 2 & No mutation \\
\hline & & 4 & c.131G>C (p.G44A) \\
\hline & & 12 & c.131G>T (p.G44V) \\
\hline
\end{tabular}

\section{Discussion}

MED12 mutation is the most common mutation in uterine leiomyomas. The mutation has been reported to vary from $40 \%$ to $85 \%$ depending on the ethnicity (Table 5). We examined the frequency of the MED12 mutations in symptomatic South Korean patients. A total of 60 leiomyomas from 41 patients were studied, and $66.67 \%(40 / 60)$ harbored a MED12 mutation. Comparing to the studies of Asian countries, this is higher than the frequency of previous Korean and Chinese studies and lower than that of the Japanese study [10-13].

In this study, multiple uterine leiomyomas seemed to have more MED12 mutations than single uterine leiomyomas did $(72.73 \%$ versus $59.26 \%)$, but there was no significant difference $(p=0.270)$. And the mean size of leiomyomas was smaller in patients with multiple leiomyomas significantly, which appears to be in correspondence with the outcome of previous study [6]. In 2015, Osinovskaya et al. demonstrated the difference of MED12 mutation frequency between multiple and single uterine leiomyomas from 122 patients [14]. The frequency of MED12 mutation was almost two-folds higher in the multiple uterine leiomyomas than in the single uterine leiomyomas, significantly ( $61 \%$ versus $32.5 \%$, $\mathrm{p}=0.003)$. However, they could not confirm the significant association between MED12 mutation and tumor size. Therefore, larger sample size of study will be needed to evaluate the association between MED12 mutation frequency and the number or the size of uterine leiomyoma.

Table 5. Frequency of the MEDI2 stations in leiomyoma in various ethnicities.

\begin{tabular}{|c|c|c|c|c|}
\hline Reference & Year & Nationality & Ethnicity & Frequency \\
\hline Heinonen et al. [6] & 2014 & Finland & Caucasian & $85.5 \%(65 / 76)$ \\
\hline Makinen et al. [5] & 2011 & Finland & Caucasian & $70.6 \%(159 / 225)$ \\
\hline McGuire et al. [8] & 2012 & USA & $\begin{array}{l}\text { Black American } \\
\text { White American }\end{array}$ & $\begin{array}{l}78 \%(18 / 23) \\
66 \%(79 / 120)\end{array}$ \\
\hline Je et al. [10] & 2012 & Korea & Asian & $52.2 \%(35 / 67)$ \\
\hline Bertsch et al. [25] & 2014 & USA & $\begin{array}{l}\text { Black women } \\
\text { White women } \\
\text { Hispanic women } \\
\text { Asian women }\end{array}$ & $\begin{array}{l}79.0 \%(64 / 81) \\
71.6 \%(53 / 73) \\
81.3 \%(13 / 16) \\
66.7 \%(4 / 6)\end{array}$ \\
\hline Makinen et al. [9] & 2011 & South Africa & $\begin{array}{l}\text { Black South } \\
\text { African and } \\
\text { Coloured }\end{array}$ & $50 \%(14 / 28)$ \\
\hline Matubara et al. [11] & 2013 & Japan & Asian & $80 \%(36 / 45)$ \\
\hline Ye et al. [12] & 2015 & China & Asian & $54.39 \%(93 / 171)$ \\
\hline Wu et al. [13] & 2017 & China & Asian & $43.6 \%(158 / 362)$ \\
\hline $\begin{array}{l}\text { Osinovskaya et al. } \\
\text { [14] }\end{array}$ & 2016 & Russia & Russian & $51.5 \%(63 / 122)$ \\
\hline
\end{tabular}

Of 14 patients with multiple leiomyomas, 9 patients harbored different types of MED12 mutations for each leiomyoma or had mutation-positive and -negative leiomyomas concurrently. Among the patients, 2 patients who had 3 masses showed different types respectively, which suggests that multiple leiomyomas may have arose from separate origin and they may not be caused by intrauterine metastasis or dissemination. The result shows that diverse factors may influence on the generation of leiomyoma and each lesion of multiple leiomyoma might have different genetic mutation. And we found some multiple leiomyomas had the same mutation types, which we could not exclude the possibility of concurrent same mutation. We considered sampling errors might have occurred but in the patient cases with multiple leiomyomas, we had extracted each tissue from definitely different tumors so we could 
exclude the errors.

MED12 mutation is also detected in other uterine tumors such as leiomyosarcomas $(30 \%)$ and smooth muscle tumor of uncertain malignant potential $(8 \%)$ but not in other organs' tumors [15-17]. Je et al. reported that among 1,862 tumor tissues including a variety of carcinomas, leukemias and stromal tumors, $52.2 \%(35 / 67)$ of uterine leiomyomas and $0.3 \%$ $(1 / 389)$ of colon carcinoma harbored MED12 mutations [10]. Another study which examined uterine leiomyosarcoma and colorectal cancer showed similar results $(7 \%, 0.5 \%)$ [18]. Interestingly, breast fibroadenoma harbored highly frequent MED12 mutations [19]. No genes except MED12 mutation were found in MED12 mutation-positive and -negative leiomyomas by whole exome sequencing and this suggests that MED12 mutation alone may be sufficient for leiomyoma tumorigenesis [20].

Recently, some studies have attempted to reveal the function of MED12 mutation in leiomyoma pathogenesis. Di et al. examined MED 12 mutation in uterine leiomyoma, myometrium and pseudocapsule. The mutation was harbored only in leiomyoma tissues. They also detected that high level of IGF-2 mRNA when MED12 missense mutations were expressed [21]. Kämpjärvi et al. examined exon 1 and exon 2 MED12 mutations in total 611 samples of uterine leiomyosarcomas, extrauterine leiomyomas and leiomyosarcomas, endometrial polyps, and colorectal cancers. All of these tumors harbored both exon 1 and exon 2 mutations, despite significantly higher rates of exon 2 mutations. Also they observed that MED12 mutations disrupt the interaction between MED12 and Cyclin C, CDK8/19 and interrupt the mediator-associated CDK kinase activity [22]. Previous studies have reported the interaction between MED12 and $\beta$-catenin/Wnt pathway [5, 23]. However, according to Perot et al., there was no association between MED12 mutations and $\beta$-catenin localization [24].

Throughout the study, we identified high frequency of the MED12 mutation in uterine leiomyomas of South Korean patients. We also identified various MED12 mutation status in multiple leiomyoma. This suggests that in a given patient, different tumors may have arisen from different cell origins and therefore it is supposed that occurrence of multiple leiomyoma in a single patient may not be caused by intrauterine metastasis or dissemination.

\section{Acknowledgments}

This research was supported by Basic Science Research Program through the National Research Foundation of Korea (NRF) funded by the Ministry of Science, ICT \& Future Planning (2012R1A1A3020083) and the Ministry of Education (2017R1D1A1 B03028045). In addition, we'd like to thank Jee Yune Park for English proofreading.

\section{Ethical approval}

All procedures performed in studies involving human participants were in accordance with the ethical standards of the institutional and/or national research committee and with the 1964 Helsinki declaration and its later amendments or comparable ethical standards.

\section{Informed consent}

Informed consent was obtained from all individual participants included in the study.

\section{Competing Interests}

The authors have declared that no competing interest exists.

\section{References}

1. Catherino WH, Parrott E, Segars J. Proceedings from theNational Institute of Child Health and Human Development conference on the Uterine Fibroid Research Update Workshop. Fertil Steril. 2011; 95: 9-12.

2. Flake GP, Andersen J, Dixon D. Etiology and pathogenesis of uterine leiomyomas: a review. Environ Health Perspect. 2003; 111: 1037-54.

3. Sandberg AA. Updates on the cytogenetics and molecular genetics of bone and soft tissue tumors: leiomyoma. Cancer genetics and cytogenetics. 2005; 158: 1-26.

4. Parker WH. Etiology, symptomatology, and diagnosis of uterine myomas. Fertil Steril. 2007; 87: 725-36.

5. Makinen N, Mehine M, Tolvanen J, Kaasinen E, Li Y, Lehtonen HJ, et al. MED12, the mediator complex subunit 12 gene, is mutated at high frequency in uterine leiomyomas. Science. 2011; 334: 252-5.

6. Heinonen HR, Sarvilinna NS, Sjöberg J, Kämpjärvi K, Pitkänen E, Vahteristo P, et al. MED12 mutation frequency in unselected sporadic uterine leiomyomas. Fertil Steril. 2014; 102: 1137-42.

7. Mehine M, Kaasinen E, Mäkinen N, Katainen R, Kämpjärvi K, Pitkänen E, et al. Characterization of uterine leiomyomas by whole-genome sequencing. The New England journal of medicine. 2013; 369: 43-53.

8. McGuire MM, Yatsenko A, Hoffner L, Jones M, Surti U, Rajkovic A. Whole exome sequencing in a random sample of North American women with leiomyomas identifies MED12 mutations in majority of uterine leiomyomas. PloS one. 2012; 7: e33251.

9. Mäkinen N, Heinonen HR, Moore S, Tomlinson IP, van der Spuy ZM, Aaltonen LA. MED12 exon 2 mutations are common in uterine leiomyomas from South African patients. Oncotarget. 2011; 2: 966-9.

10. Je EM, Kim MR, Min KO, Yoo NJ, Lee SH. Mutational analysis of MED12 exon 2 in uterine leiomyoma and other common tumors. International journal of cancer Journal international du cancer. 2012; 131: E1044-7.

11. Matsubara A, Sekine S, Yoshida M, Yoshida A, Taniguchi H, Kushima R, et al. Prevalence of MED12 mutations in uterine and extrauterine smooth muscle tumours. Histopathology. 2013; 62: 657-61.

12. Ye J, Wang $\mathrm{H}$, Chen YB, Yuan DL, Zhang LX, Liu L. MED12 mutation in patients with hysteromyoma. Oncology letters. 2015; 9: 2771-4.

13. Wu J, Zou Y, Luo Y, Guo JB, Liu FY, Zhou JY, et al. Prevalence and clinical significance of mediator complex subunit 12 mutations in 362 Han Chinese samples with uterine leiomyoma. Oncology letters. 2017; 14: 47-54.

14. Osinovskaya NS, Malysheva OV, Shved NY, Ivashchenko TE, Sultanov IY, Efimova OA, et al. Frequency and Spectrum of MED12 Exon 2 Mutations in Multiple Versus Solitary Uterine Leiomyomas From Russian Patients. International journal of gynecological pathology : official journal of the International Society of Gynecological Pathologists. 2016; 35: 509-15.

15. Schwetye KE, Pfeifer JD, Duncavage EJ. MED12 exon 2 mutations in uterine and extrauterine smooth muscle tumors. Human pathology. 2014; 45: 65-70.

16. Ravegnini G, Mariño-Enriquez A, Slater J, Eilers G, Wang Y, Zhu M, et al. MED12 mutations in leiomyosarcoma and extrauterine leiomyoma. Modern pathology : an official journal of the United States and Canadian Academy of Pathology, Inc. 2013; 26: 743-9.

17. Mäkinen N, Vahteristo $P$, Kämpjärvi $K$, Arola J, Bützow R, Aaltonen LA. MED12 exon 2 mutations in histopathological uterine leiomyoma variants. European journal of human genetics : EJHG. 2013; 21: 1300-3. 
18. Kämpjärvi K, Mäkinen N, Kilpivaara O, Arola J, Heinonen HR, Böhm J, et al. Somatic MED12 mutations in uterine leiomyosarcoma and colorectal cancer. British journal of cancer. 2012; 107: 1761-5.

19. Lim WK, Ong CK, Tan J, Thike AA, Ng CC, Rajasegaran V, et al. Exome sequencing identifies highly recurrent MED12 somatic mutations in breast fibroadenoma. Nature genetics. 2014; 46: 877-80.

20. Mäkinen N, Vahteristo P, Bützow R, Sjöberg J, Aaltonen LA. Exomic landscape of MED12 mutation-negative and -positive uterine leiomyomas. International journal of cancer Journal international du cancer. 2014; 134: 1008-12.

21. Di Tommaso S, Tinelli A, Malvasi A, Massari S. Missense mutations in exon 2 of the MED12 gene are involved in IGF-2 overexpression in uterine leiomyoma. Molecular human reproduction. 2014; 20: 1009-15.

22. Kämpjärvi $K$, Park $M J$, Mehine $M$, Kim NH, Clark $A D$, Butzow $R$, et al Mutations in Exon 1 highlight the role of MED12 in uterine leiomyomas. Human mutation. 2014; 35: 1136-41.

23. Markowski DN, Bartnitzke S, Löning T, Drieschner N, Helmke BM, Bullerdiek J. MED12 mutations in uterine fibroids--their relationship to cytogenetic subgroups. International journal of cancer Journal international du cancer. 2012; 131: 1528-36.

24. Pérot G, Croce S, Ribeiro A, Lagarde P, Velasco V, Neuville A, et al. MED12 alterations in both human benign and malignant uterine soft tissue tumors. PloS one. 2012; 7: e40015.

25. Bertsch E, Qiang W, Zhang Q, Espona-Fiedler M, Druschitz S, Liu Y, et al. MED12 and HMGA2 mutations: two independent genetic events in uterine leiomyoma and leiomyosarcoma. Modern pathology : an official journal of the United States and Canadian Academy of Pathology, Inc. 2014; 27: 1144-53. 